\title{
Implementing genetic screening for the management of hepatic disease
}

\author{
Brittany Dewdney, Lionel Hebbard \\ Department of Molecular and Cell Biology, College of Public Health, Medical, and Veterinary Sciences, James Cook University, Townsville, \\ Australia \\ Correspondence to: Dr. Lionel Hebbard. Department of Molecular and Cell Biology, James Cook University, Townsville, Australia. \\ Email: lionel.hebbard@jcu.edu.au. \\ Provenance: This is an invited Editorial commissioned by Editor-in-Chief Yilei Mao (Department of Liver Surgery, Peking Union Medical College \\ Hospital, Chinese Academy of Medical Sciences, Beijing, China). \\ Comment on: Zhang DY, Goossens N, Guo J, et al. A hepatic stellate cell gene expression signature associated with outcomes in hepatitis C cirrhosis \\ and hepatocellular carcinoma after curative resection. Gut 2016;65:1754-64.
}

Submitted Jun 29, 2017. Accepted for publication Jul 13, 2017.

doi: $10.21037 /$ hbsn.2017.07.06

View this article at: http://dx.doi.org/10.21037/hbsn.2017.07.06

Liver cirrhosis is an advanced stage of liver fibrosis causing liver failure, and may progress to hepatocellular carcinoma (HCC). HCC remains as the second leading cause of cancer-related deaths worldwide and is one of few cancer types to show increasing incidence and mortality rates (1). HCC incidence has doubled in low-risk countries such as USA, Canada, Australia, and some European countries since the 1970s (2) and mortality rates have increased in these regions, especially in $45+$ age groups (3). This may be attributed to intravenous drug use in this cohort associated with high HCV infection rates between 1960-1980 (4). Additionally, an increasing amount of HCC cases are nonviral (5). Thus, the rise in HCC deaths in Westernized countries may be attributed to increased obesity and diabetes prevalence, both well-established risk factors for non-alcoholic fatty liver disease (NAFLD), non-alcoholic hepatic steatosis (NASH), and progression to HCC. Regardless of original cause, $85-90 \%$ of HCC cases arise from underlying cirrhosis and non-cirrhotic cases show varying stages of fibrosis. Unfortunately, progression of hepatic fibrosis to cirrhosis is often asymptomatic and most diagnoses are not made until clinical signs arise indicating end-stage liver disease (ESLD) or advanced HCC (6). Thus, a relevant strategy of reducing liver disease-related deaths are to take preventative measures and improve disease management.

A study has recently been published that may address the clinical need for improved management strategies of cirrhosis progression. Zhang and colleagues identified a gene signature unique to hepatic stellate cells (HSC) that is correlated to prognosis of cirrhosis-related liver disease (7). HSCs are one of the primary initiators of fibrosis development. Liver injury induces HSC activation and differentiation into myofibroblasts that produce extracellular matrix (ECM) proteins (8). ECM remodelling and accumulation of ECM secreted proteins generates fibrosis, which may progress to cirrhosis if chronic injury sustains HSC proliferation and fibrogenic signalling (8). Zhang and colleagues used 98 murine datasets from 17 liver cell types to identify a unique subset of genes specifically expressed in activated HSCs (7). The 122-gene signature was used to develop a prognostic index for predicting clinical outcomes in two patient cohorts characterized by early cirrhosis. The prognostic index was first derived in HCV-infected patients with early stage (Child-Pugh class A) cirrhosis $(n=216)$ to assess overall survival (OS), hepatic decompensation, progression of Child-Pugh class, and HCC development. The validation cohort included curatively resected early stage HCC patients $(n=82)$ in which OS and clonally independent recurrence of HCC were examined. Multivariable Cox regression analysis demonstrated the HSC gene signature to be independently associated with increased progression of Child-Pugh class ( $\mathrm{HR}=2.16)$ and decreased survival $(\mathrm{HR}=2.34)$ in the $\mathrm{HCV}$ 
cohort, after adjustment for standard clinical prognostic characteristics (serum bilirubin $>1 \mathrm{mg} / \mathrm{dL}$ and platelet count $<100,000 \mathrm{~mm}^{3}$ ). The novel Cox regression model including the HSC signature improved the prognostic index of the clinical model (serum bilirubin and platelet count alone) in predicting death (0.66 to 0.70$)$ and progression of ChildPugh class (0.68 to 0.70 ) in $\mathrm{HCV}$ patients and in predicting death (0.58 to 0.62) in HCC patients. Predictive prognosis models require a c-statistic of 0.70 or greater for clinical utility. Therefore, the HSC gene signature can only be used as a prognostic marker for the aforementioned clinical outcomes in the HCV cohort, though the practicality of the signature within these settings is unclear. The addition of other clinical characteristics to the model, such as patient age and serum albumin, may enhance the prognostic value of the HSC gene signature.

The HSC gene signature showed no association with $\mathrm{HCC}$ development or HCC recurrence in the $\mathrm{HCV}$ cohort and HCC cohort, respectively, nor did it improve the prognostic index for either outcomes in these cohorts. This is expected as the fibrotic microenvironment is vastly different from the tumour microenvironment. Although fibrosis and cirrhosis are strong risk factors for HCC, progression to tumour growth will occur through a cellular switch to oncogenic signalling. The majority of the genes within the HSC signature are involved in organ development, cell differentiation, and ECM production, thus will not likely significantly contribute to such a transformation.

Further advances by these authors have implicated another gene signature specific to risk of HCC development (9). This 186-gene set is enriched in genes associated with poor outcome and are involved in increased cell cycle signalling, cell growth and increased proliferative signalling (9), thus are more strongly correlated with a tumour-promoting environment. Interestingly, only 3 genes are common to the HSC signature and the HCC risk signature. ITGA9, LOXL2, and TGB1I1 were identified by Zhang and colleagues as genes with the highest correlation to poor prognosis within the 122-gene set (7). ITGA9 and TGFB1I1 are involved in cytoskeletal remodelling to promote epithelial-mesenchymal transition (EMT), are often induced in fibrosis $(10,11)$. Similarly, LOXL2 contributes to ECM remodelling and is a potent inducer of invasion in cancer cells (12). While these genes are implicated in multiple types of malignant cancers, it is reasonable to expect upregulation of these genes in fibrotic phenotypes. ITGA9, LOXL2, and TGFB1I1 may serve as valuable prognostic markers for fibrosis progression, however, without co-expression of other oncogenic genes contributing to the signature they are unlikely to be predictive of HCC development. Therefore, the significance of the HSC gene signature may not lie in prognostic value, but rather in future therapeutic developments to suppress fibrosis.

Gene set enrichment analysis demonstrated high HSC gene signature expression in human cirrhotic liver [normalised enrichment score (NES) 2.08; false discovery rate (FDR) $<0.001$ ], NAFLD patient liver with severe fibrosis (NES 1.97; FDR 0.002), and NAFLD patients with inflammation (NES 1.49; FDR 0.071), when compared to their respective control groups, non-cirrhotic, NAFLD with moderate fibrosis, and NAFLD with no inflammation. Interactive pathway analysis revealed significant involvement of transforming growth factor $\beta$ (TGF- $\beta$ ), platelet derived growth factor receptor (PDGFR), and ERK1/2 in regulation of the signature genes within all disease groups. This highlighted the common pathways involved in HSC activation and identified unique downstream targets for anti-fibrotic therapy, including ITGA9, LOXL2, and TGFB1I1. Previous studies have examined LOXL2 as a therapeutic target, however, showed no benefit in reducing fibrosis in patients (13). Interestingly, a pre-clinical study demonstrated inhibition of liver fibrosis in TGFB1I1-knock out models (14). Therefore, inhibitory drug cocktails targeting LOXL2 and TGFB1I1 may be more efficient in suppressing fibrosis progression. Nonetheless, this HSC signature provides a unique opportunity for researchers to hand-pick clinically relevant genes from a predetermined collection of fibrosis promoting targets, allowing for development of general and patient-specific therapeutic strategies.

Interestingly, in rodent fibrosis models the HSC gene signature was enriched in mouse/rat bile duct ligation models, mouse unilateral urethral obstruction models, and rat diethylnitrosamine models, but not in carbon tetrachloride $\left(\mathrm{CCl}_{4}\right)$ mouse or rat models. Though $\mathrm{CCl}_{4}$ treatment is one of the most commonly employed hepatic fibrosis models in rodents, it results in significantly lower serum bilirubin and higher platelet counts in comparison to other hepatoxic agents (15). Considering high bilirubin $(>1 \mathrm{mg} / \mathrm{dL})$ and low platelet count $\left(<100,000 / \mathrm{mm}^{3}\right)$ is the clinical baseline indicative of liver failure, $\mathrm{CCl}_{4}$-induced fibrotic models may not be suitable in terms of clinical translation to human fibrosis. Furthermore, $\mathrm{CCl}_{4}$ fibrosis models demonstrated little upregulation of liver TGF- $\beta$ expression (15), however, increased TGF- $\beta$ is characteristic 
of human fibrosis-related diseases and HCC. Zhang and colleagues confirmed high expression of TGF- $\beta$ in HSCs in cirrhosis patients, NAFLD patients with severe fibrosis, and NAFLD patients with inflammation (7). Therefore, this study emphasizes the clinical utility of various animal models of fibrosis. Adjustments in pre-clinical studies will further improve future therapeutic investigations.

Furthermore, Zhang and colleagues identified a novel HSC cell surface marker, PCDH7. PCDH7 showed significant expression in human and murine HSCs compared to hepatocytes and endothelial cells (7). While yet to be confirmed, the authors implicate $\mathrm{PCDH} 7$ as a protocadherin likely involved in cell-cell interactions and intracellular signalling. Future opportunities lie in characterizing the function of this protein and implicating $\mathrm{PCDH} 7$ in HSC isolation techniques and HSC-targeted therapy.

This study systematically derived a unique gene set specific to HSCs in human fibrosis-related diseases. Although the gene signature is strongly enriched in cirrhotic and fibrotic conditions and is associated with decreased survival in HCV and HCC patients, improvements in the prognostic index are required before it can be employed as an accurate model for predicting clinical outcomes. Despite this, the HSC signature has been deemed a valuable tool not only for implementing novel therapeutic targets, but also as a tool for monitoring fibrosis progression. In a subsequent study the authors demonstrated suppression of the HSC gene signature in human fibrotic livers treated ex vivo with inhibitors of the lysophosphatidic acid (LPA) pathway to block downstream expression of pro-cirrhotic genes (9). However, additional considerations need to be made regarding the feasibility of incorporating a liver gene signature into clinical measures, especially during early stages of disease. Invasive and expensive techniques would be required to isolate this information from patients. Compressed versions of the signature (31- and 55-genes) demonstrated similar or improved associations with clinical outcomes in both patient cohorts (7), which may reduce the cost associated with sample analyses. These gene sets should be implemented further in future therapeutic interventions for close monitoring and management of liver disease progression.

\section{Acknowledgements}

Funding: This work was supported by Cancer Council NSW Project Grants (1069733, L Hebbard), Cancer
Council Queensland Project Grant (1123436, L Hebbard) and a James Cook Development Grant 2016 (L Hebbard).

\section{Footnote}

Conflicts of Interest: The authors have no conflicts of interest to declare.

\section{References}

1. Hashim D, Boffetta P, La Vecchia C, et al. The global decrease in cancer mortality: trends and disparities. Ann Oncol 2016;27:926-33.

2. Petrick JL, Braunlin M, Laversanne M, et al. International trends in liver cancer incidence, overall and by histologic subtype, 1978-2007. Int J Cancer 2016;139:1534-45.

3. Bertuccio P, Turati F, Carioli G, et al. Global trends and predictions in hepatocellular carcinoma mortality. J Hepatol 2017;67:302-9.

4. Ryerson AB, Eheman CR, Altekruse SF, et al. Annual Report to the Nation on the Status of Cancer, 1975-2012, Featuring the Increasing Incidence of Liver Cancer. Cancer 2016;122:1312-37.

5. El-Serag HB. Hepatocellular carcinoma: recent trends in the United States. Gastroenterology 2004;127:S27-34.

6. Heidelbaugh JJ, Bruderly M. Cirrhosis and chronic liver failure: part I. Diagnosis and evaluation. Am Fam Physician 2006;74:756-62.

7. Zhang DY, Goossens N, Guo J, et al. A hepatic stellate cell gene expression signature associated with outcomes in hepatitis $\mathrm{C}$ cirrhosis and hepatocellular carcinoma after curative resection. Gut 2016;65:1754-64.

8. Friedman SL. Hepatic stellate cells: protean, multifunctional, and enigmatic cells of the liver. Physiol Rev 2008;88:125-72.

9. Nakagawa S, Wei L, Song WM, et al. Molecular Liver Cancer Prevention in Cirrhosis by Organ Transcriptome Analysis and Lysophosphatidic Acid Pathway Inhibition. Cancer Cell 2016;30:879-90.

10. Gupta SK, Oommen S, Aubry MC, et al. Integrin $\alpha 9 \beta 1$ promotes malignant tumor growth and metastasis by potentiating epithelial-mesenchymal transition. Oncogene 2013;32:141-50.

11. Wu JR, Hu CT, You RI, et al. Hydrogen peroxide inducible clone- 5 mediates reactive oxygen species signaling for hepatocellular carcinoma progression. Oncotarget 2015;6:32526-44.

12. Wu $\mathrm{L}, \mathrm{Zhu} \mathrm{Y}$. The function and mechanisms of action of 
LOXL2 in cancer (Review). Int J Mol Med 2015;36:1200-4.

13. Meissner EG, McLaughlin M, Matthews L, et al.

Simtuzumab treatment of advanced liver fibrosis in HIV and $\mathrm{HCV}$-infected adults: results of a 6-month open-label safety trial. Liver Int 2016;36:1783-92.

14. Lei XF, Fu W, Kim-Kaneyama JR, et al. Hic-5 deficiency attenuates the activation of hepatic stellate cells and liver

Cite this article as: Dewdney B, Hebbard L. Implementing genetic screening for the management of hepatic disease. HepatoBiliary Surg Nutr 2017;6(5):359-362 . doi:10.21037/ hbsn.2017.07.06 fibrosis through upregulation of Smad7 in mice. J Hepatol 2016;64:110-7.

15. Park HJ, Kim HG, Wang JH, et al. Comparison of TGF-beta, PDGF, and CTGF in hepatic fibrosis models using DMN, CCl4, and TAA. Drug Chem Toxicol 2016;39:111-8. 